\title{
INTERFERÊNCIA DA VARIAÇÃO CAMBIAL NA AQUISIÇÃO DE MATERIAIS E NA CADEIA DE SUPRIMENTOS
}

\author{
MICHELON, Joel ${ }^{1}$ \\ REIS, Zaida Cristiane dos ${ }^{2}$ \\ BIEGELMEYAER, Uiliam $\mathrm{Hahn}^{3}$ \\ CAMARGO, Maria Emilia ${ }^{4}$ \\ CRACO, Tânia ${ }^{5}$
}

\begin{abstract}
RESUMO: No presente estudo, o objetivo principal é analisar os impactos causados pela variação cambial, no custo da aquisição de componentes para a indústria do setor Metal-Mecânico. A empresa referente ao estudo tem mais de cinquenta anos de atuação no mercado, no segmento de tratores, motores, chassi para ônibus leves e médios, caminhões leves e médios e utilitários de uso civil e militar. A metodologia de pesquisa utilizada para a elaboração do presente trabalho foi a pesquisa qualitativa descritiva com o caráter exploratório, pois teve início com o levantamento dos custos dos produtos em determinado período e posteriormente compará-los com os custos na situação atual. Devido a esse problema evidenciado na empresa, elegeu-se a gestão de estoques que está na área de compras como objeto de estudo e busca de melhorias para aumentar o desempenho da organização. Foi feito um levantamento dos processos de aquisição de materiais, onde os resultados obtidos auxiliam na identificação dos elementos que influenciam diretamente o fluxo das atividades no setor de compras. A partir daí, apresentou-se o resultado da análise, contribuindo para uma melhor gestão da área, minimizando os seus custos e apresentando os ganhos que podem ser obtidos com a devida utilização dos recursos disponíveis.
\end{abstract}

Palavras-chave: Setor Metal-Mecânico. Gestão de compras. Lead Time.

\section{INTERFERENCE OF THE EXCHANGE VARIATION ON THE ACQUISITION OF MATERIAL AND ON THE SUPPLY CHAIN}

\begin{abstract}
SUMMARY: On this study, the main goal is to analyze the impacts caused by the exchange variation on the cost of acquisition of components for the metal-mechanical industry. The company studied has been on the market for more than 50 years, actuating on the tractors, engines, frames for buses and trucks field, both civil and military. The methodology used for this work was the qualitative exploratory research, because it begun with charging the costs of the products in a certain period of time and then comparing them with the costs nowadays. Due to this problem noted on the company, the business area was chosen as an object of study and pursue of the best performance of the company. A research about the acquisition of materials was performed, and the results obtained are helping on the identification of the elements that directly influence the activity on the Business area. That being said, the result of the analysis was presented, making it better to manage the Business field, minimizing its costs and presenting the earnings that can be obtained with the correct use of the available resources.
\end{abstract}

Keywords: Metal-Mechanical Sector. Purchasing management. . Lead Time.

\section{INTRODUÇÃO}

O mercado nacional mantém-se competitivo e todos buscam alternativas para se sobressair nesta

\footnotetext{
${ }^{1}$ UCSRS - Especialista em Gestão da Produção

${ }^{2}$ UCSRS - Doutora em Administração

${ }^{3}$ PUCRS - Doutor em Administração

${ }^{4}$ UCSRS - Doutora em Engenharia de Produção

${ }^{5}$ IRFS - Farroupilha - Doutora em Administração
} 
disputa acirrada. Novas tecnologias são excelentes propulsoras de ganhos em desenvolvimento e competitividade, tanto na melhoria de produtos, como na qualidade da cadeia de fornecimento, redução dos desperdícios e busca constante de melhoria contínua de processos.

A empresa deste estudo segue as tendências mundiais, buscando inovação constante e investindo em novas tecnologias, para ser uma organização reconhecida pelas suas ações e pelos seus excelentes produtos, sempre com foco no atendimento as necessidades dos clientes, sejam eles internos ou externos.

O objetivo geral do presente trabalho é analisar os impactos causados pela variação cambial na aquisição de matéria/is oriundos do mercado externo. Para alcançá-lo foram selecionados os objetivos específicos que são a análise do lead time de entrega dos produto, a variação do câmbio no período, a programação dos materiais e também uma avaliação dos custos dos estoques.

Com base no exposto cabe apontar a seguinte questão de pesquisa: Qual é o impacto causado pela variação cambial no custo de aquisição de materiais para uma empresa do ramo metal mecânico?

\section{REFERENCIAL TEÓRICO}

Neste item serão abordados alguns tópicos que possuem interface com o tema do estudo em questão. Através das definições apresentadas, serão avaliados métodos para diminuir os custos dos materiais, problema caracterizado no objetivo deste estudo.

\subsection{Gestão de Materiais}

Segundo Viana (2012) alguns fatos foram determinantes para a confirmação e o surgimento da obrigação de que os materiais necessitam ser administrados utilizando conhecimentos específicos, onde podem ser citadas, as necessidades que as forças armadas passaram durante as duas grandes guerras mundiais, onde buscaram meios eficazes para o abastecimento de suas tropas.

Para Slack et. al. (2002) o estoque se define como a acumulação armazenada de recursos materiais em um sistema de produção.

De acordo com Gonçalves (2016) pode-se concluir que a sociedade passou a utilizar o termo gestão de materiais a partir do momento que ocorreram mudanças significativas na aérea de tecnologia. Foram incorporados softwares para auxiliar no gerenciamento dos materiais, assim como foram criados equipamentos com tecnologias extremante avançadas que auxiliam no processo produtivo dando maior agilidade para o mesmo.

Conforme Dias (2012) o objetivo principal de uma empresa é maximizar o retorno sobre o investimento, para atingir o máximo de retorno é necessário ser eficiente no planejamento e controle dos estoques. Um sistema de materiais deve estabelecer a integração desde a previsão de vendas até a produção e a entrega do produto final.

Para Martins (2011) a gestão de estoques constitui uma série de ações que permitem ao administrador verificar se os estoques estão sendo bem utilizados, bem localizados em relação aos setores que deles utilizam bem manuseados e bem controlados. Os mesmos representam uma parcela substancial dos ativos da empresa e devem ser encarados como um fator potencial de negócios e de lucros.

De acordo com Chopra (2011) uma cadeia de suprimentos engloba todos os estágios envolvidos, direta ou indiretamente no atendimento a um pedido de cliente. A cadeia de suprimentos não inclui apenas fabricantes e fornecedores, mas também transportadoras, depósitos varejistas e os próprios clientes.

Com o advento do desenvolvimento tecnológico foi possível realizar diversas melhorias no sistema de gerenciamento de materiais. Naquela época assim como hoje são necessários profissionais 
altamente capacitados para entender que a administração de materiais deve ser um dos itens do planejamento estratégico (DIAS,2012).

\subsection{Programação de Materiais}

De acordo com Gonçalves (2016) a programação de materiais com os métodos conhecidos atualmente teve seu início por volta de 1960. A programação também conhecida como MRP II tem por objetivo calcular a quantidade e os prazos de materiais necessários para a fabricação dos produtos.

O objetivo básico de gerir o estoque é de conseguir um equilíbrio dos estoques disponíveis com as demandas dos clientes, sem causar um aumento muito sensível nos custos da organização. Também é composto pelos gastos envolvidos na aquisição, conservação e manutenção efetuados principalmente na decisão de quanto e quando fazer o pedido (FERNIE, 2004).

Segundo Lélis (2016) o MRP (MaterialsRequirements Planning) estabelece uma série de procedimentos e regras para tomada de decisão com o objetivo de atender as necessidades da área de produção dentro de uma sequência de tempo. Dentre os principais, podem ser destacados a garantia de que os materiais estejam disponíveis evitando parada da produção e manutenção dos inventários no menor nível possível.

Para Gonçalves (2016) a principal dificuldade enfrentada pelo sistema na década de 1960 foi à inexistência de computadores que facilitariam a rápida realização de cálculos e em grande escala. Nesta época os cálculos eram realizados manualmente, ou seja, qualquer variação na demanda faria com que os cálculos fossem realizados novamente. Posteriormente ocorreram mudanças significativas e avanços nesta sistemática, foram introduzidos os recursos financeiros neste sistema. Mudou-se a denominação para MRP II e isso possibilitou a gestão da demanda de forma mais eficiente e abrangente. A base deste novo sistema está vinculada ao conceito de demanda independente, que é a demanda do produto final onde nela estão contidos todos os componentes para o produto.

Para Dias (2012) o MRP II tem como objetivo o planejamento da compra e produção de itens componentes para que ocorram nas quantidades e nos momentos necessários. Sua diferenciação do MRP é que o MRP II permite o gerenciamento de todos os recursos da empresa incluindo nesta análise os recursos financeiros.

Posterior à avaliação da programação dos materiais é necessária uma análise do tempo de ressuprimento dos itens necessários, cabe então o estudo do Lead Time.

\subsection{Lead Time}

O modelo econômico tem conduzido o mercado a exigir além de uma qualidade maior, uma quantidade de produtos igualmente grande e um custo menor, mas sobre tudo em intervalo de tempo bem diminutos. As empresas, para serem competitivas, focam em velocidade de produção (MELLO, et al,2016).

Segundo Baily (2011) o tempo de entrega dos materiais é um fator estratégico para as empresas, visto que quanto menor o tempo para o recebimento dos materiais, menor será o prazo de entrega do produto ao cliente, que é um dos objetivos das empresas.

Conforme Gonçalves (2016) é o tempo de ressuprimento, que representa o espaço de tempo decorrido entre o momento da manifestação da necessidade de um material até sua disponibilidade para o consumo. O Lead time está diretamente relacionado com a diminuição de investimentos em estoque, redução de custos operacionais aumentando assim a margem de lucro das empresas. 
Para Baily (2011) é através do Lead time que é possível assegurar as entregas de materiais necessários serão feitas para atender as programações de produção. Uma vez que a estocagem pode ser vista como um seguro contra a falta de suprimentos, teoricamente as programações de entregas perfeitas eliminariam os estoques. Ainda este autor afirma que para buscar vantagem competitiva e preparar-se melhor para responder as necessidades dos clientes a medida que elas surgem é natural que seja exigido um maior grau de responsabilidade dos fornecedores.

O autor destaca que a entrega a tempo é o objetivo de compras, se os materiais chegarem atrasados ou o trabalho não for concluído no tempo correto as vendas podem ser perdidas. Diante das teorias acima verificadas pode-se concluir que o Lead time é um fator estratégico para a empresa. O Lead time não é nada mais que o tempo necessário da verificação da necessidade de certos materiais até o momento que o mesmo encontra-se disponível para sua utilização no processo fabril. Cabe aos compradores fazer desta estratégia um diferencial para a empresa, assim antecipando-se a concorrência.

\subsection{Custo dos Estoques}

De acordo com Gonçalves (2016) o estoque de materiais é fundamental para a organização, é através dele que as empresas atendem a seus planos de produção com maior agilidade e menor tempo de entrega. Por outro lado, também é o fator que mais agrega custos na empresa, os materiais em estoque significam recursos que poderiam ser investidos em outras áreas estão armazenados em forma de matéria prima ou componentes.

Gonçalves (2016) descreve ainda que quando tratam-se de estoques tem-se sempre dois pontos de vista, um deles é o financeiro que para este o estoque significa imobilizado da empresa. Para o departamento de logística isso significa custos de armazenagens e movimentação.

Conforme Dias (2012) é importante medir o quanto, em reais a empresa tem investido em estoque, e quanto isso representa em custo, em comparação com o montante das vendas, produção efetiva e previsão das vendas. Quanto menor for este valor melhor para a empresa.

Segundo Chopra (2011) o estoque é a antecipação da demanda, seu papel é aumentar a quantidade de demanda a ser atendida. O estoque é o principal fator gerador de custos na cadeia de suprimentos e exerce um enorme impacto no poder de resposta da empresa. Por outro lado, um estoque alto pode representar um menor tempo de fluxo entre a entrada do pedido até o atendimento ao cliente. Para conseguir resultados positivos é importante estabelecer acordos de longo prazo envolvendo estoques mínimos compartilhando informações e conhecimentos de maneira a atingir soluções rápidas para os problemas enfrentados no cotidiano da organização (FERREIRA, LI, SERRA, 2010).

Através do controle e monitoramento dos custos dos estoques, com uso de ferramentas e indicadores é possível efetuar uma análise crítica e traçar estratégias para atingir os objetivos da organização (BONASSINA et al,2018).

De acordo com Francischini (2013) os custos dos estoques podem ser divididos em custo de aquisição, custo de armazenagem, custo de pedido e custo de falta. O custo de aquisição é o valor pago pela empresa compradora pelo material adquirido. $\mathrm{O}$ custo de armazenagem é aquele oriundo do material dentro da empresa, sabe-se quanto mais tempo em estoque a mercadoria permanece em estoque maior será seu custo. Já o custo de pedido é o valor gasto pela empresa para que determinado lote de compra possa ser solicitado ao fornecedor e entregue na empresa compradora. E por último tem-se o custo de falta, é aquele custo que é difícil de ser calculado com precisão, uma vez que envolve uma série de estimativas, rateios e valores intangíveis, porém pode causar prejuízos à empresa.

Segundo Viana (2012) o custo de comprar é descrito através da seguinte formula: 
Figura 1 - Fórmula do Custo de Comprar

\begin{tabular}{|l|c|l|}
\hline \multirow{2}{*}{$\mathrm{CC}=$} & VALOR R $\$$ & $\mathrm{CC}=$ CUSTO DE COMPRAR \\
\cline { 2 - 3 } & ITENS & VALOR R $\$=$ VALOR DE AQUISIÇÃO \\
\hline \multirow{2}{*}{} & ITENS = QUANTIDADE DE ITENS \\
\hline
\end{tabular}

Fonte: Adaptado de Viana, 2012.

Já o custo de armazenagem é descrito através da seguinte fórmula:

Figura 2 - Fórmula do Custo de Armazenagem

\begin{tabular}{|c|c|l|}
\hline \multirow{2}{*}{$\mathrm{CA}=$} & $12 \mathrm{X} \mathrm{R} \$$ & \multicolumn{1}{c|}{ CA = CUSTO DE ARMAZENAR } \\
\cline { 2 - 3 } & $\mathrm{K}$ & VALOR R $\$=$ VALOR DE AQUISIÇÃO \\
\hline & $\mathrm{K}=$ QTD DE ITENS EM ESTOQUE \\
\hline
\end{tabular}

Fonte: Adaptado de Viana,2012.

E por fim, o custo total dos materiais é descriminado pela seguinte fórmula:

Figura 3 - Fórmula do Custo Total do Estoque

\begin{tabular}{|l|l|}
\hline \multirow{4}{*}{$\mathrm{CT}=\mathrm{CC}+\mathrm{CA}$} & $\mathrm{CT}=\mathrm{CUSTO}$ TOTAL DO ESTOQUE \\
\cline { 2 - 2 } & $\mathrm{CC}=$ CUSTO DE COMPRAR \\
\cline { 2 - 2 } & $\mathrm{CA}=$ CUSTO DE ARMAZENAR \\
\hline
\end{tabular}

Fonte: Adaptado de Viana, 2012.

Após a análise dos impactos nas finanças da empresa pelos materiais armazenados, resta o procedimento de aquisição propriamente dito, assunto tratado na sequência.

\subsection{Compras}

De acordo com Gonçalves (2016) as aquisições de materiais nas empresas representam mais de $50 \%$ do custo total. Devido a este fato a função requer um bom planejamento e também execução. Para isso a empresa necessita de profissionais altamente capacitados e com experiência no assunto. A função de compras sofre com a economia globalizada, onde os mercados são factíveis as pressões políticas e econômicas e as variações cambiais afetam os custos de aquisição, por isso muitas empresas optam apenas pela cadeia nacional de fornecimento, porem pode ocorrer a escassez de materiais.

Conforme Dias (2012) compras é um segmento essencial e tem a finalidade de suprir as necessidades de materiais ou serviços, no momento certo, com as quantidades corretas e providenciar seu armazenamento. Além disso, têm-se os objetivos básicos de manter o fluxo continuo de suprimentos para atender os programas de produção, ou ainda, comprar materiais e insumos com menores preços, obedecendo aos padrões de qualidade e quantidade definidos.

Segundo Gonçalves (2016) compra é o ato e a responsabilidade para promover a procura dos materiais e dos serviços a serem utilizados na empresa. A função de compras envolve uma responsabilidade muito maior, onde requer planejamento, acompanhamento, monitoramento e análise para que o produto seja recebido no momento esperado, nas quantidades desejadas e na qualidade solicitada. 
Conforme Martins (2011) hoje a função de compras é vista como parte do processo de logística das empresas, ou seja, parte integrante da cadeia de suprimentos, por isso as empresas passaram a usar a denominação gerenciamento da cadeia de suprimentos. A área de compras compete ainda o cuidado com os níveis de estoque da empresa, pois altos níveis de estoque acarretam um custo exagerado para sua manutenção.

De acordo com Baily (2011) o processo de compras é uma função chave na administração de uma empresa, pois quando considerado o custo total de aquisição de materiais e serviços requer uma análise do que está sendo especificado em relação a aquilo que o mercado oferta. Esta área tem um extremo potencial na redução de custos e consequentemente em retorno dos investimentos da empresa.

De acordo com Viana (2012) o ato de comprar inclui etapas como a determinação de quanto e quando comprar, um estudo dos potenciais fornecedores e a verificação de sua capacidade técnica, o fechamento do pedido mediante autorização de fornecimento ou contrato, acompanhamento durante o período de pedido até a entrega e o encerramento do processo após recebimento do material com o controle da qualidade e quantidade.

Viana (2012) descreve ainda que comprar é uma arte das mais antigas, por este motivo exige que o comprador possua qualificações, demonstrando conhecimentos dos procedimentos, das características dos materiais, assim como ser bom negociador que é fundamental. $\mathrm{O}$ comprador deve ter responsabilidade e autoridade, ter iniciativa, capacidade de decisão, objetividade e idoneidade, essas são as principais características que norteiam sua conduta profissional.

\subsection{Variação Cambial}

De acordo com Eiteman (2013) o mercado de câmbio fornece a estrutura física internacional onde a moeda de um país é trocada pela moeda de outro país. A taxa de câmbio entre as moedas é determinada e as transações de câmbio são fisicamente completadas. A transação de câmbio é um contrato entre um comprador e um vendedor o qual a quantia de uma moeda deve ser entregue a uma taxa específica em troca de outra moeda.

Conforme Eiteman (2013) o mercado de câmbio é o mecanismo pelo qual se transferem poderes de compra entre países, obtendo ou fornecendo crédito para transações de negócio internacionais e minimizando a exposição aos riscos de mudança das taxas de câmbio. A taxa de câmbio é o preço de uma moeda expressa em termos de outra moeda. Uma cotação de câmbio é a demonstração de disposição de comprar ou vender a uma taxa anunciada.

\subsection{Modelo Conceitual de Análise}

Segue no Quadro 1 as principais ideias apresentadas no referencial teórico, nele estão descritos alguns conceitos dos autores referentes a gestão de materiais e através deles pode-se evidenciar a importância do assunto na administração das empresas. 
Quadro 1 - Principais conceitos

\begin{tabular}{|c|c|c|}
\hline Ideias & Autores & Ano \\
\hline $\begin{array}{l}\text { A sociedade passou a utilizar o termo gestão de materiais a partir do momento } \\
\text { que ocorreram mudanças significativas na aérea de tecnologia. Foram } \\
\text { incorporados softwares para auxiliar no gerenciamento dos materiais. }\end{array}$ & Gonçalves & 2016 \\
\hline $\begin{array}{l}\text { O objetivo principal de uma empresa é maximizar o retorno sobre o } \\
\text { investimento. Um sistema de materiais deve estabelecer a integração desde a } \\
\text { previsão de vendas até a produção e a entrega do produto final. }\end{array}$ & Dias & 2012 \\
\hline $\begin{array}{l}\text { O MRP (MaterialsRequirements Planning) estabelece uma série de } \\
\text { procedimentos e regras para tomada de decisão com o objetivo de atender as } \\
\text { necessidades da área de produção dentro de uma sequência de tempo. }\end{array}$ & Lélis & 2016 \\
\hline $\begin{array}{l}\text { O Lead time representa o espaço de tempo decorrido entre o momento da } \\
\text { manifestação da necessidade de um material até sua disponibilidade para o } \\
\text { consumo o mesmo está diretamente relacionado com a diminuição de } \\
\text { investimentos em estoque, redução de custos e etc. }\end{array}$ & Gonçalves & 2016 \\
\hline $\begin{array}{l}\text { O estoque é o principal fator gerador de custos na cadeia de suprimentos e } \\
\text { exerce um enorme impacto no poder de resposta da empresa. Por outro lado, } \\
\text { um estoque alto pode representar um menor tempo de fluxo entre a entrada do } \\
\text { pedido até o atendimento ao cliente. }\end{array}$ & Chopra & 2011 \\
\hline $\begin{array}{l}\text { O processo de compras é uma função chave na administração de uma empresa, } \\
\text { pois quando considerado o custo total de aquisição de materiais e serviços } \\
\text { requer uma análise do que está sendo especificado em relação a aquilo que o } \\
\text { mercado oferta. }\end{array}$ & Baily & 2011 \\
\hline
\end{tabular}

Fonte: Adaptado pelos autores.

Com base nas ideias dos autores apresentadas anteriormente, será apresentado a análise dos dados e o roteiro de entrevista que foi fundamentado nas ideias deste quadro 1. Na sequência será descrita a metodologia utilizada na elaboração deste artigo.

\section{METODOLOGIA}

Para a elaboração deste trabalho foi utilizada técnica de pesquisa qualitativa descritiva. A primeira técnica que pode ser citada é a observação ,realizada nas dependências da empresa, pois foi através dela que se descobriu a existência de um problema. Verificar as variações de produtos adquiridos no mercado externo e a variação de preços em função da oscilação cambial. No segundo momento podem ser citadas as entrevistas, não estruturadas, mas sim de maneira a coletar as informações iniciais, sem roteiro definido com os envolvidos, colaboradores que possuem vínculo com a área em estudo. Estes foram de extrema importância pois conseguiram compartilhar suas experiências na área e mais especificamente com o assunto abordado. Cabe salientar que o entrevistador possui sólidos conhecimentos na área abrangida pela entrevista como gestão de estoques , materiais, lead time, taxa cambial, entre outras que foram indagadas 
aos entrevistados. E por último foi realizada a análise documental dos checklist dos embarques e comparados ao custo cadastrado dos produtos na data.

Dentre as técnicas de coleta de dados existentes cabe salientar que foram utilizadas ainda a coleta documental, observação em loco e análise de indicadores. Os dados coletados são de caráter quantitativo. O processo de coleta de dados foi continuamente acompanhado pelo elaborador deste estudo.

\section{DESENVOLVIMENTO DA PESQUISA}

Nos anos de 2015 e 2016 ocorreu um constante acréscimo do valor de cotação do dólar, com isso os custos de importação de componentes acabam se tornando maiores. A empresa em estudo importa grande parte de seus produtos, assim os valores de aquisição ficaram expressivos. Faz parte de um grupo do segmento metal mecânico renomado na Região da serra Gaúcha, composto por quatro unidades no Brasil e uma na Argentina. Ela é diferenciada por atender as mais diversas demandas de seus clientes, desde motores, tratores, caminhões, chassis até veículos militares, através da versatilidade de seus projetos e processos produtivos, sempre utilizando tecnologia adequada a cada produto. Atualmente a empresa conta com 1100 funcionários distribuídos em suas quatro unidades no cenário nacional.

$\mathrm{Na}$ realização deste artigo foram coletadas informações com pessoas experientes da área em estudo. Dentre os colaboradores podem ser citados; Supervisor de Compras, Gerente de Pós-vendas e ainda o Gerente de Engenharia.

O processo de coleta dos dados ocorreu através da verificação dos custos do produto no ano de 2014 em relação aos custos do produto no ano de 2016 utilizando-se de uma análise documental junto a este departamento. Estes dados foram coletados junto ao departamento de planejamento da empresa e no departamento de compras.

Pode-se evidenciar através dos dados coletados que no início do ano de 2014 o dólar estava cotado em $\mathrm{R} \$ 2,38$ e assim foram analisadas as médias mensais do mesmo chegando em dezembro com o valor de $\mathrm{R} \$ 2,63$ ou seja, houve um acréscimo de $\mathrm{R} \$ 0,25$ no período que corresponde a um percentual de $10 \%$.

Já o ano de 2015 inicia-se com o dólar a $\mathrm{R} \$ 2,63$ e chega-se no mês de dezembro com o mesmo cotado a $\mathrm{R} \$ 3,87$, ou seja, ocorre um aumento de $\mathrm{R} \$ 1,24$ e isso representa um percentual de $47 \%$.

Em 2016, iniciou-se com $\mathrm{R} \$ 4,05$ em janeiro e no mês de junho fechou em $\mathrm{R} \$ 3,42$, ou seja, temse uma queda de $\mathrm{R} \$ 0,63$ o que representa $16 \%$ de variação.

Deste modo, verifica-se a variação durante todo o período de trinta meses chegando a seguinte variação. De R \$ 2,38 em janeiro de 2014 para R \$ 3,42 em junho de 2016 obtendo uma diferença de R\$ 1,04 ou $30 \%$.

É possível ainda uma análise mais profunda no contexto. Pode-se analisar os extremos, como por exemplo o mês de maio de 2014 quando a cotação do dólar era $\mathrm{R} \$ 2,22$ e relaciona-se com o mês de janeiro de 2016 quando a média foi $R \$ 4,05$ tem-se uma diferença de $R \$ 1,83$ que representa um percentual de $55 \%$ de variação.

Trazendo estes dados para a realidade da empresa é possível afirmar que um produto cuja participação na aquisição é $100 \%$ do mercado externo o custo do mesmo no período irá variar em média $30 \%$ positivamente, ou seja, irá custar para a empresa $30 \%$ a mais do que o previsto.

$\mathrm{Na}$ sequência verificam-se alguns destes dados coletados. Na Tabela 1, pode-se realizar um acompanhamento das variações da moeda Dólar (EUA) em relação a moeda nacional o Real. Este levantamento foi realizado no período de janeiro de 2014 até o mês de junho de 2016, ou seja, nos últimos trinta meses. 
Tabela 1 - Variação do Dólar

\begin{tabular}{r|l|r|r|r|r}
\hline \multicolumn{1}{c|}{ DATA } & MOEDA & COMPRA & VENDA & Média Compra & Média Venda \\
\hline $31 / 01 / 2014$ & USD & 2,4257 & 2,4263 & 2,3816 & 2,3822 \\
\hline $28 / 02 / 2014$ & USD & 2,3327 & 2,3334 & 2,3837 & 2,3837 \\
\hline $31 / 03 / 2014$ & USD & 2,2624 & 2,263 & 2,3255 & 2,3261 \\
\hline $30 / 04 / 2014$ & USD & 2,2354 & 2,236 & 2,2322 & 2,2328 \\
\hline $30 / 05 / 2014$ & USD & 2,2384 & 2,239 & 2,2203 & 2,2209 \\
\hline $30 / 06 / 2014$ & USD & 2,2019 & 2,2025 & 2,2349 & 2,2355 \\
\hline $31 / 07 / 2014$ & USD & 2,2668 & 2,2674 & 2,224 & 2,2246 \\
\hline $29 / 08 / 2014$ & USD & 2,239 & 2,2396 & 2,2674 & 2,2680 \\
\hline $30 / 09 / 2014$ & USD & 2,4504 & 2,451 & 2,3323 & 2,3329 \\
\hline $31 / 10 / 2014$ & USD & 2,4436 & 2,4442 & 2,4476 & 2,4483 \\
\hline $28 / 11 / 2014$ & USD & 2,5595 & 2,5601 & 2,5477 & 2,5484 \\
\hline $31 / 12 / 2014$ & USD & 2,6556 & 2,6562 & 2,6387 & 2,6394 \\
\hline $30 / 01 / 2015$ & USD & 2,6617 & 2,6623 & 2,6336 & 2,6342 \\
\hline $27 / 02 / 2015$ & USD & 2,8777 & 2,8782 & 2,8158 & 2,8165 \\
\hline $31 / 03 / 2015$ & USD & 3,2074 & 3,208 & 3,1389 & 3,1395 \\
\hline $30 / 04 / 2015$ & USD & 2,993 & 2,9936 & 3,0452 & 3,0458 \\
\hline $29 / 05 / 2015$ & USD & 3,1781 & 3,1788 & 3,0611 & 3,0617 \\
\hline $30 / 06 / 2015$ & USD & 3,1019 & 3,1026 & 3,1111 & 3,1117 \\
\hline $31 / 07 / 2015$ & USD & 3,3934 & 3,394 & 3,2225 & 3,2231 \\
\hline $31 / 08 / 2015$ & USD & 3,6461 & 3,6467 & 3,5137 & 3,5143 \\
\hline $30 / 09 / 2015$ & USD & 3,9722 & 3,9729 & 3,9058 & 3,9065 \\
\hline $30 / 10 / 2015$ & USD & 3,8582 & 3,8589 & 3,8795 & 3,8801 \\
\hline $30 / 11 / 2015$ & USD & 3,8499 & 3,8506 & 3,7758 & 3,7765 \\
\hline $31 / 12 / 2015$ & USD & 3,9042 & 3,9048 & 3,8705 & 3,8711 \\
\hline $29 / 01 / 2016$ & USD & 4,0422 & 4,0428 & 4,0517 & 4,0524 \\
\hline $29 / 02 / 2016$ & USD & 3,979 & 3,9796 & 3,9731 & 3,9737 \\
\hline $30 / 03 / 2016$ & USD & 3,611 & 3,6116 & 3,7102 & 3,7108 \\
\hline $29 / 04 / 2016$ & USD & 3,4502 & 3,4508 & 3,5652 & 3,5658 \\
\hline $31 / 05 / 2016$ & USD & 3,5945 & 3,5951 & 3,5387 & 3,5393 \\
\hline $30 / 06 / 2016$ & USD & 3,2092 & 3,2098 & 3,4239 & 3,4245 \\
\hline & & FD & & \\
\hline
\end{tabular}

Fonte: Adaptado pelos autores.

Na Tabela 2 pode-se evidenciar a diferença de custo de um dos produtos adquiridos pela empresa na pratica. Este produto é oriundo do mercado asiático e sua aquisição ocorre em dólares. 
Tabela 2 - Variação nos custos da cabine

\begin{tabular}{|c|c|c|c|c|c|}
\hline \multicolumn{6}{|c|}{ VARIAÇÃO NO VALOR FOB - CABINE AAAAA } \\
\hline$\#$ & Data & Cotação USD & USD1,00 & Cotação BRL & Motivo da variação \\
\hline 1 & $\mathrm{abr} / 14$ & $\$ \quad 2.870,00$ & $\mathrm{R} \$ 2,24$ & $\mathrm{R} \$ \mathbf{6} \quad 6.428,80$ & Cotação \\
\hline 2 & ago/14 & $3.100,00$ & $\mathrm{R} \$ 2,27$ & $7.037,00$ & Cotação negociada \\
\hline 3 & out/14 & $3.429,63$ & $\mathrm{R} \$ \mathbf{2} 2,45$ & $8.402,59$ & PI primeira ordem, acréscimo de componentes. \\
\hline 4 & nov/14 & $3.429,63$ & $\mathrm{R} \$ 2,55$ & $8.745,56$ & PI segunda ordem \\
\hline 5 & $\mathrm{jan} / 15$ & $3.439,85$ & $\mathrm{R} \$ 2,63$ & $\mathrm{R} \$ \quad 9.046,81$ & PI terceira ordem, acréscimo dos faróis de neblina. \\
\hline 6 & $\mathrm{mar} / 15$ & $3.439,85$ & $\mathrm{R} \$ 3,30$ & $\mathrm{R} \$ 11.351,51$ & Atualização cambial \\
\hline 7 & jun/15 & $3.439,85$ & $\mathrm{R} \$ 3,06$ & $\mathrm{R} \$ 10.525,94$ & Atualização cambial \\
\hline 8 & nov/15 & $3.439,85$ & $\mathrm{R} \$ 3,78$ & $\mathrm{R} \$ 13.002,63$ & Atualização cambial \\
\hline 9 & jan/16 & $3.439,85$ & $\mathrm{R} \$ \quad 4,05$ & $\mathrm{R} \$ 13.931,39$ & Atualização cambial \\
\hline 10 & jun/16 & $3.439,85$ & $\mathrm{R} \$ 3,42$ & $\mathrm{R} \$ 11.764,29$ & Atualização cambial \\
\hline
\end{tabular}

Como pode ser verificado o produto que no mês de abril de 2014 custava para a empresa R \$ $6.428,80$, no mês de janeiro de 2015 passou a custar R $\$ 9.046,81$, ou seja, $29 \%$ a mais. Esta diferença nos custos dificilmente poderá ser passada ao cliente na íntegra.

Na Figura 4 é possível evidenciar a crescente nos custos do produto ocasionada pela variação cambial. Esta imagem refere-se ao mesmo produto acima demonstrado, mesmo fornecedor e também com a mesma moeda.

Figura 4 - Variação nos custos do produto

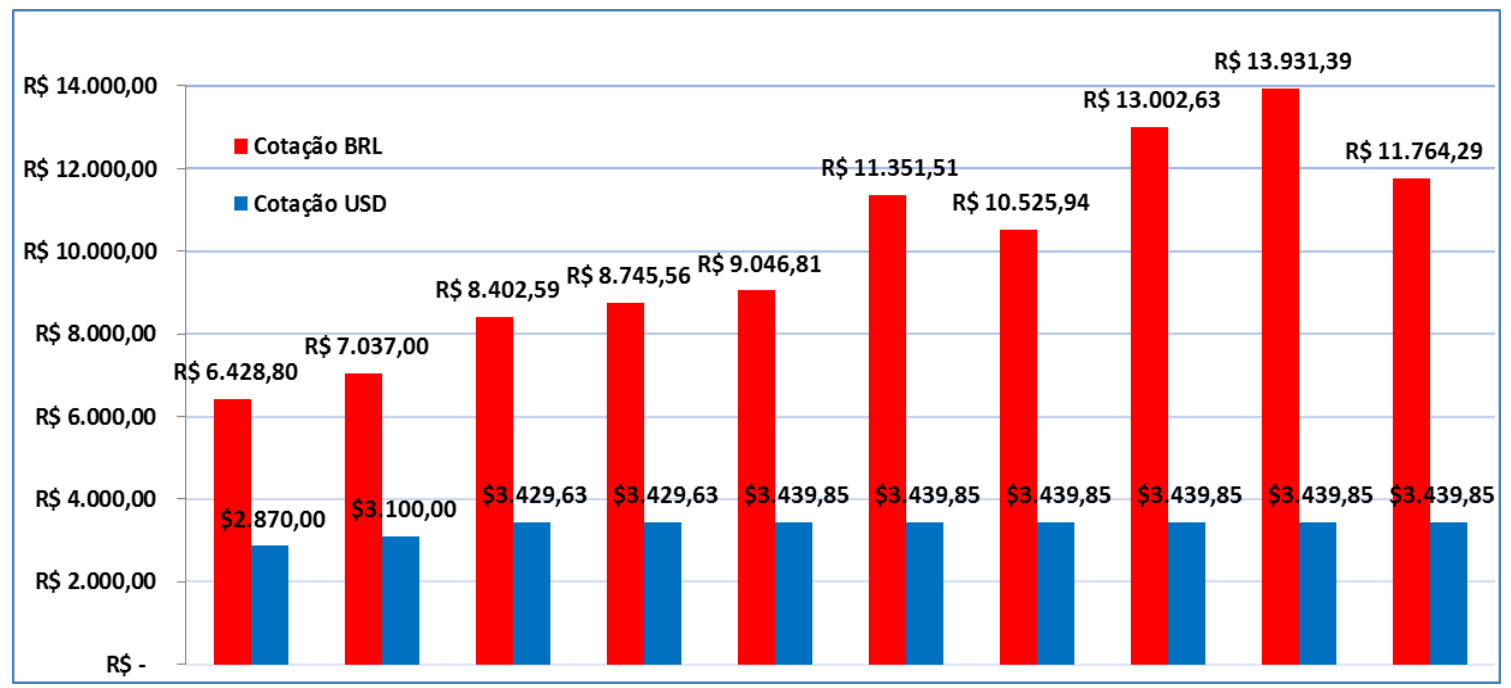

Fonte: Adaptado pelo autor.

Na Figura 5 pode-se evidenciar a crescente variação cambial durante o período em estudo. Durante os trinta meses estudados em apenas dois momentos houve queda em comparação com o período anterior. 
Figura 5 - Variação Dólar X Real

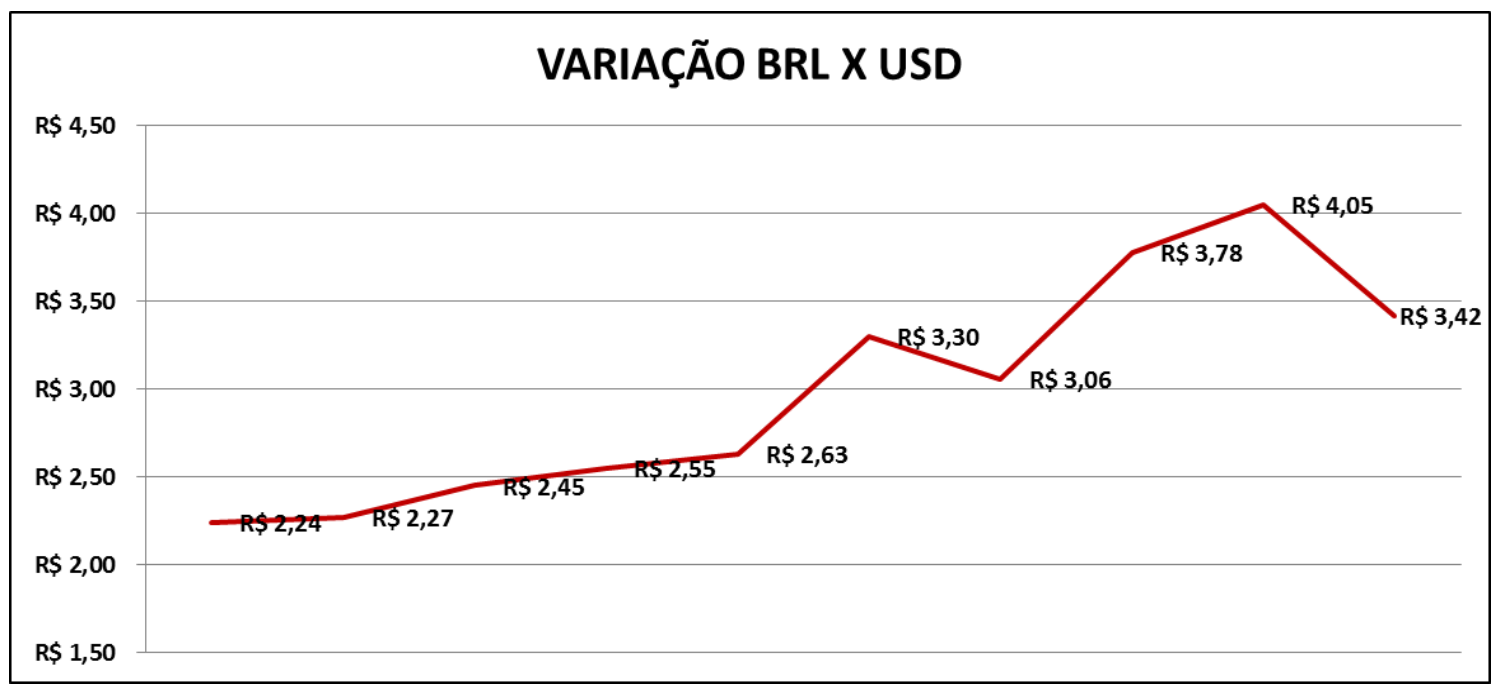

Fonte: Adaptado pelo autor

Na Tabela 3 pode-se evidenciar a diferença de custo de mais um dos produtos adquiridos pela empresa. Este produto é oriundo do mercado asiático e sua aquisição ocorre em dólares.

Tabela 3 - Variação nos custos do eixo dianteiro

VARIAÇÃO NO VALOR FOB - EIXO TRASEI RO C/ FREIOS AAAAA

\begin{tabular}{|c|c|c|c|c|c|c|c|c|}
\hline \multirow{2}{*}{$\frac{\#}{1}$} & \multirow{2}{*}{\begin{tabular}{|l} 
Data \\
$a b r / 14$
\end{tabular}} & \multicolumn{2}{|c|}{ Cotação USD } & \multicolumn{2}{|c|}{ USD1,00 } & \multicolumn{2}{|c|}{ Cotação BRL } & \multirow{2}{*}{\begin{tabular}{|l} 
Motivo da variação \\
Cotação
\end{tabular}} \\
\hline & & $\$$ & $1.137,00$ & $\mathrm{RS}$ & 2,24 & $\mathrm{RS}$ & $2.546,88$ & \\
\hline 2 & ago/14 & $\$$ & 851,76 & $\mathrm{R} \$$ & 2,27 & $\mathrm{RS}$ & $1.933,50$ & Cotação negociada \\
\hline 3 & out/14 & $\$$ & 909,56 & $\mathrm{R} \$$ & 2,45 & $\mathrm{R} \$$ & $2.228,42$ & PI primeira ordem \\
\hline 4 & nov/14 & $\$$ & 909,56 & $\mathrm{R} \$$ & 2,55 & $\mathrm{R} \$$ & $2.319,38$ & PI segunda ordem \\
\hline 5 & $\mathrm{jan} / 15$ & $\$$ & 909,56 & $\mathrm{R} S$ & 2,63 & $\mathrm{R} \$$ & $2.392,14$ & PI terceira ordem \\
\hline 6 & mar/15 & $\$$ & 909,56 & $\mathrm{R} \$$ & 3,14 & $\mathrm{RS}$ & $2.856,02$ & Atualização cambial \\
\hline 7 & jun/15 & $\$$ & 909,56 & $\mathrm{R} \$$ & 3,06 & $\mathrm{R} \$$ & $2.783,25$ & Atualização cambial \\
\hline 8 & nov/15 & $\$$ & 909,56 & $\mathrm{R} \$$ & 3,78 & $\mathrm{RS}$ & $3.438,14$ & Atualização cambial \\
\hline 9 & $\mathrm{jan} / 16$ & $\$$ & 909,56 & $\mathrm{R} \$$ & 4,05 & $\mathrm{RS}$ & $3.683,72$ & Atualização cambial \\
\hline 10 & jun/16 & $\$$ & 909,56 & $\mathrm{RS}$ & 3,42 & $\mathrm{RS}$ & $3.110,70$ & Atualização cambial \\
\hline
\end{tabular}

Fonte: Adaptado pelo autor

Na Figura 6 é possível evidenciar a crescente nos custos do produto, o eixo dianteiro com freios e molas ocasionado pela variação cambial. 
Figura 6 - Alteração no custo devido a variação cambial

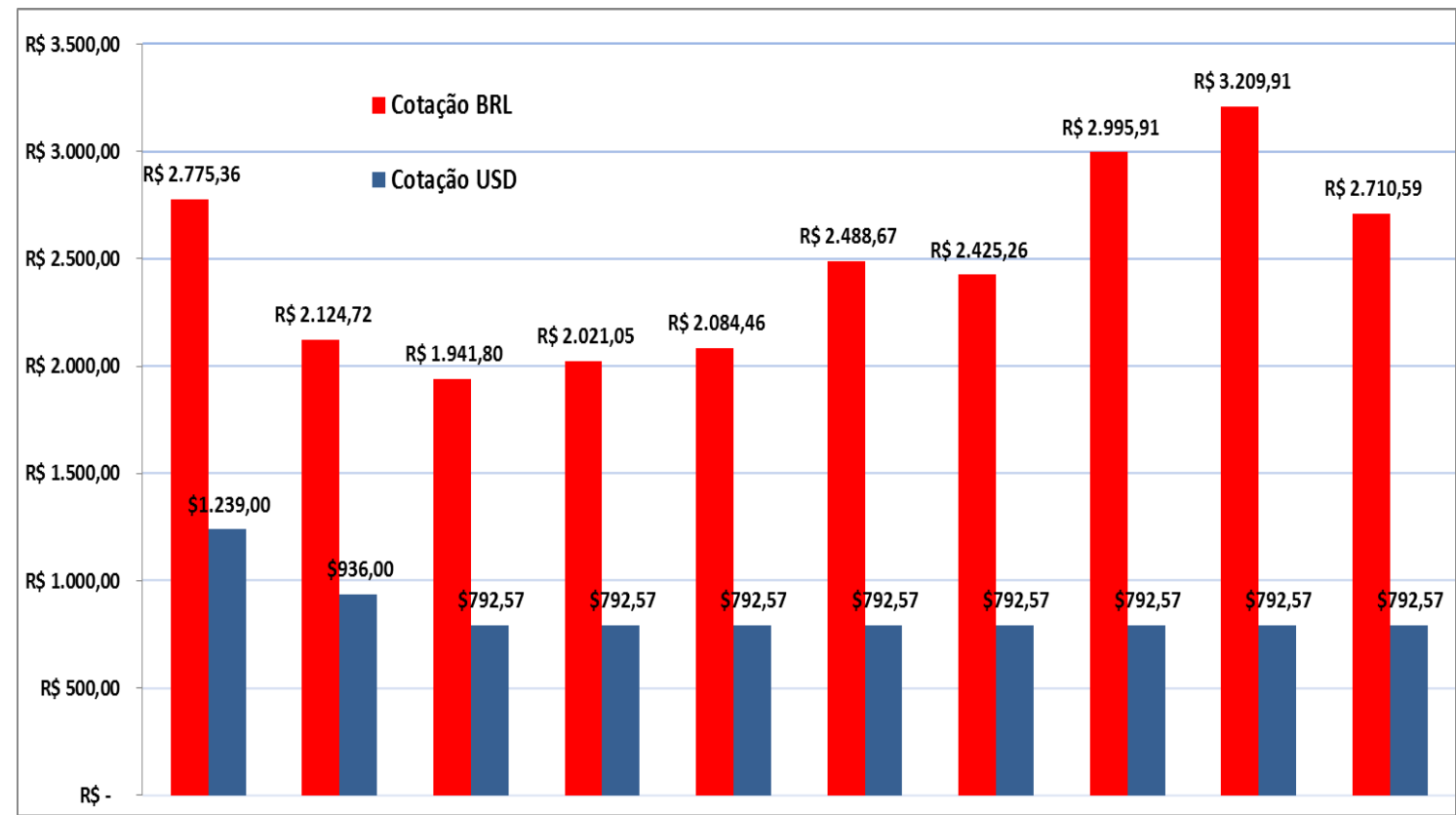

Fonte: Adaptado pelo autor

$\mathrm{Na}$ Tabela 4 é possível evidenciar a diferença de custo de um dos produtos adquiridos pela empresa. Neste caso tem-se o eixo traseiro com os freios, oriundos do mercado asiático e com a aquisição em dólares.

Tabela 4 - Variação nos custos do eixo traseiro

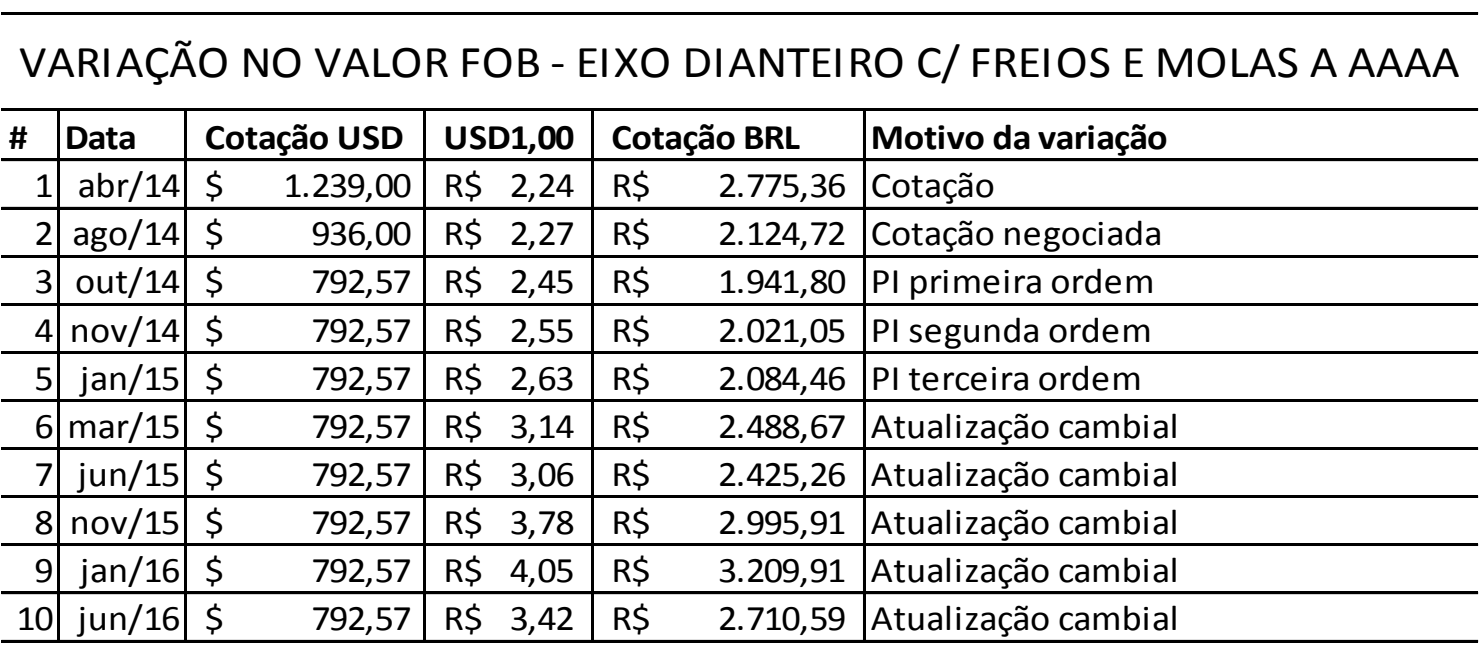

Fonte: Adaptado pelo autor 
Na Figura 7 pode-se evidenciar a crescente ocasionada nos custos do produto pela variação cambial. Houve na maioria dos períodos uma manutenção das cotações porem com a variação do dólar os custos aumentaram drasticamente.

Figura 7 - Alteração no custo devido a variação cambial

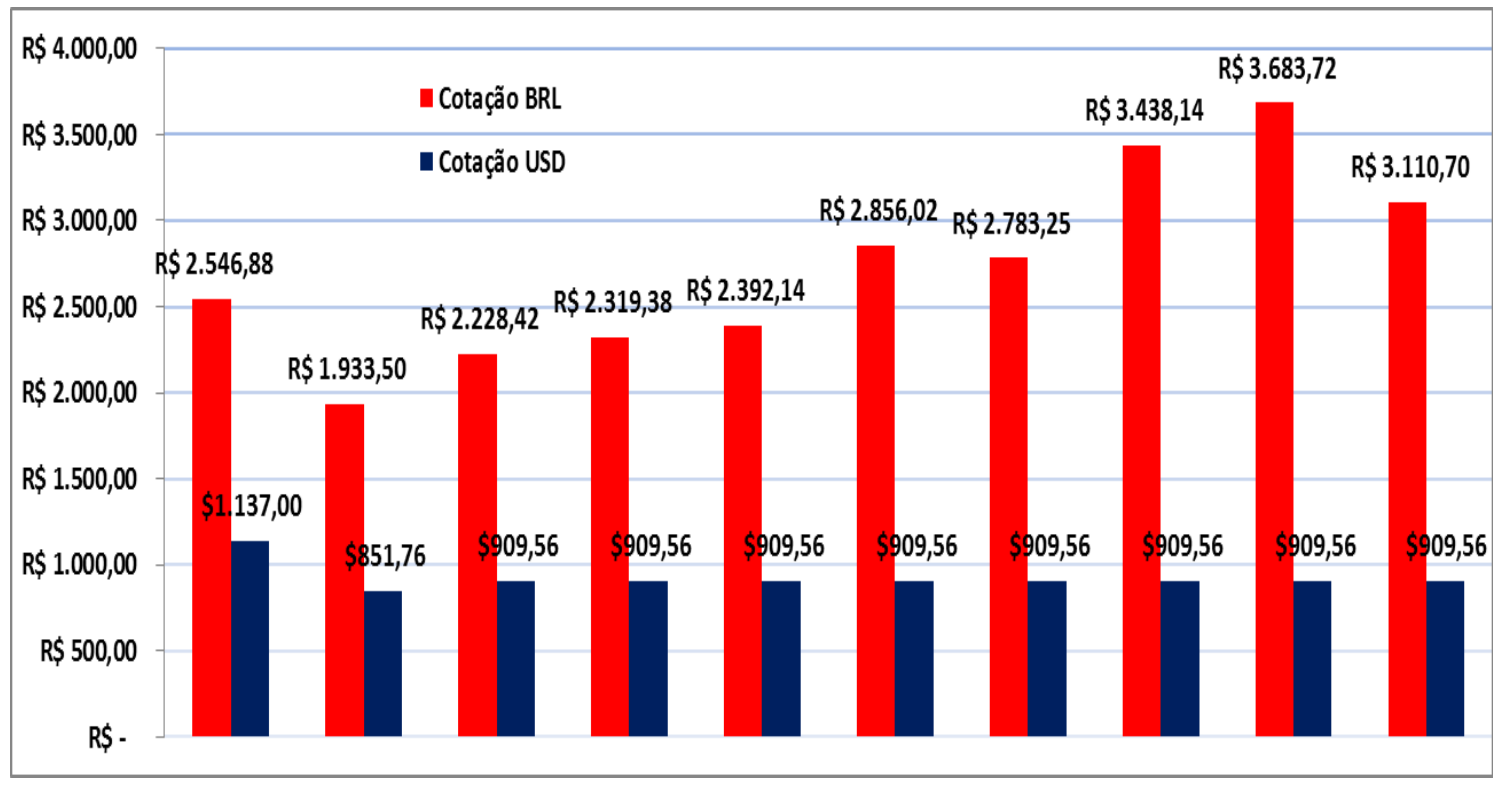

Fonte: Adaptado pelo autor

A análise dos dados ocorreu através de gráficos de custos atuais em comparação com o ano de 2014. Quando finalizado o trabalho de coleta e análise, foi realizado um comparativo entre a situação atual e a situação anterior ao aumento do câmbio.

O objetivo do estudo com base na análise do cenário atual busca indicar os impactos causados por esta variação, apontando as perdas de capital e vendas ocasionadas por este processo. A Tabela 4auxilia na análise dos impactos.

Tabela 5 - Variação Cambial e Variação nos Custos dos Itens

\begin{tabular}{|c|c|c|c|c|c|c|c|c|c|}
\hline \multicolumn{6}{|c|}{ VARIAÇÃO CAMBIAL - RESUMO } & \multicolumn{4}{|c|}{ VARIAÇÃO NOS CUSTOS CABINE -RESUMO } \\
\hline ANO & \multicolumn{2}{|c|}{ JAN } & \multicolumn{2}{|c|}{ DEZ } & \# & ANO & 2014 & 2016 & $\#$ \\
\hline 2014 & $\mathbf{R} \$$ & 2,38 & $\mathbf{R} \mathbf{S}$ & 2,63 & $10.00 \%$ & JAN & $\mathrm{R} \$ \mathbf{7 . 7 0 5 , 2 6}$ & & \multirow{2}{*}{$65 \%$} \\
\hline 2015 & $\mathbf{R} \$$ & 2,63 & $\mathbf{R} \$$ & 3,87 & $47.00 \%$ & JUN & & $\mathrm{R} \$ 11.764,29$ & \\
\hline 2016 & $\mathbf{R} \$$ & 4,05 & $\mathbf{R} \$$ & 3,42 & $16.00 \%$ & \multicolumn{2}{|c|}{ COTAÇÃO ATUAL } & $\$$ & $3.439,85$ \\
\hline \# TOTAL & $\mathbf{R} \$$ & 2,38 & $\mathbf{R} \mathbf{S}$ & 3,42 & $30.00 \%$ & NEGOC. & $\$ 2.870,00$ & $3.439,85$ & $\$-569,85$ \\
\hline \multicolumn{6}{|c|}{ VARIAÇÃO NOS CUSTOS EIXO DIANT -RESUMO } & \multicolumn{4}{|c|}{ VARIAÇÃO NOS CUSTOS EIXO TRAS -RESUMO } \\
\hline ANO & \multicolumn{2}{|c|}{2014} & \multicolumn{2}{|c|}{2016} & $\#$ & ANO & 2014 & 2016 & $\#$ \\
\hline JAN & $\mathrm{R} \$$ & 774,08 & & & $52 \%$ & JAN & $\mathrm{R} \$ 2.036,16$ & & \multirow{2}{*}{$53 \%$} \\
\hline JUN & & & \multicolumn{2}{|c|}{$\mathrm{R} \$ 2.708,64$} & סנדב & JUN & & $\mathrm{R} \$ \mathbf{3} \quad 3.108,78$ & \\
\hline \multicolumn{3}{|c|}{ COTAÇÃO ATUAL } & $\$$ & & 792,00 & \multicolumn{2}{|c|}{ COTAÇÃO ATUAL } & $\mathrm{R} \$$ & 909,00 \\
\hline NEGOC. & $\$$ & $.239,00$ & $\$$ & 792,00 & $\$ 447,00$ & NEGOC. & $\$ 1.137,00$ & 909,00 & $\$ 228,00$ \\
\hline
\end{tabular}

Fonte: Adaptado pelo autor 
Como se pode evidenciar na Tabela 4, o custo dos componentes aumentou em média 53\% devido as alterações solicitadas e também a variação cambial. O projeto foi iniciado com um dólar a $\mathrm{R} \$ 2,24$ (abril de 2014) e no mês de junho de 2016 fechou em $\mathrm{R} \$ 3,42$. A diferença nos custos não foi maior devido às negociações entre as empresas.

Através dos dados analisados, constata-se que nem sempre a importação é uma vantagem competitiva, para a empresa em estudo isso fez com que aumentasse drasticamente os custos dos seus produtos vendidos. Esses custos dificilmente poderão ser repassados ao cliente na integra.

Nem sempre se consegue alcançar os objetivos propostos, pois estas oscilações cambiais podem comprometer a rentabilidade e muitas vezes até mesmo oferecer um grande risco à sustentabilidade da própria organização no que diz respeito à continuidade da mesma (BONASSINA et al:;2018).

\section{CONCLUSÃO}

O processo de importação sofre interferências de alguns riscos, atrasos, avarias, e variação cambial. Isto pode ser minimizado com um planejamento e um bom processo de importação (PICININ,KOVALESKI,PEDROSO, 2011).

Nenhuma nação é autossuficiente em tudo, que não necessite importar algo. A importação tem vantagens, como contribuir com o crescimento econômico do país em um cenário de globalização. É um fator estratégico para a empresa que importa, como o baixo custo da aquisição, o tempo da importação pode ser menor que o tempo necessário para produzir internamente, algum incentivo governamental, o menor nível e mão de obra necessário e a própria variação cambial favorável (BRIDI et al;.2014).

A importação apresenta muitos desafios, principalmente para as micro e pequenas empresas, mas ao mesmo tempo muitas oportunidades que podem contribuir para a competitividade das organizações.

No mercado atual de grande competitividade e inovação as empresas buscam renovar seus produtos e processos, à medida que as organizações se dedicam para atingir seus objetivos devem estar cientes que ocorrem grandes movimentações internas ou externas. A empresa em estudo se destaca por atuar em segmentos que exigem muita agilidade e rapidez para atingir os requisitos e a satisfação dos clientes.

Concluir que a importação pode não ser uma estratégia que sempre é a mais assertiva. Para o caso em questão isso causou prejuízos ou aumento dos seus custos. Mas foi um caso específico. Cada operação deve ser avaliada de maneira isolada. O que pode não ser viável para este ítem em questão, talvez seja solução para outro componente, máquina ou insumo que a organização necessite. Para diminuir os impactos a empresa deverá aumentar a quantidade de itens exportados para tentar equilibrar a balança comercial.

Como sugestão para a empresa de metalurgia estudada seria importante desenvolver um fornecedor nacional para poder dispor de uma alternativa que não ficasse totalmente atrelada à variação cambial.

Este estudo de caso foi limitado à empresa em questão e poderá ser transmitido as empresas do mesmo segmento como modelo, desde que observados que os custos de materiais estejam de acordo com a fonte de fornecimento e ainda de acordo com a moeda vigente na fonte de fornecimento. 


\section{REFERENCIAS}

BANCO CENTRAL DO BRASIL. Disponível em:

http://www4.bcb.gov.br/pec/taxas/port/ptaxnpesq.asp?id=txcotacao Acesso em: 03 set. 2016.

BAILY, P.et al. Compras: princípios e administração. São Paulo: Atlas, 2011.

BONASSINA,D.et al. The Importance of Logistics Costs for Organizations Applied to Logistics and Supply Chain TroughsSustainable Management. European Journal of Scientific Research, v 150,p 299310,2018 .

BRIDI,C.D. et al. Application of the RBV as a Tool for Obtaining Competitive Advantages in a Metallurgic Company. Australian Journal Of Basic And Applied Sciences. v. 8,n.9, p. 340-350, 2014.

CHOPRA, S. Gestão da cadeia de suprimentos: estratégia, planejamento e operações. 4 ed. São Paulo: Pearson Prentice Hall, 2011. Acesso em: 09 set.2016.

DIAS, M.A.P. Administração de materiais: princípios, conceitos e gestão. 6.ed. São Paulo: Atlas, 2012.

EITEMAN, D.K. Administração Financeira Internacional. 12. ed. Porto Alegre: Bookman, 2013.

FERNIE,J. Retail Logistics. In: BRUCE, M; MOORE, C; BIRTWISTLE,G. International Retailer Marketing: a Case Study Approach. Oxford, Ma Elsevier Butterworth- Heinemann, 2004

FERREIRA, M. P.; LI, D.; SERRA, F. A. R. Transferência internacional de conhecimento na multinacional: quando o jogo competitivo multimercado se sobrepõe aos mecanismos internos de coordenação. RAE, v. 9, n. 1, 2010.

FRANCISCHINI, P.G.; GURGEL, F.A. Administração de Materiais e do Patrimônio. 2. ed. São Paulo: Cengage Learning, 2013.

GONÇALVES, P.S. Administração de Materiais. 3.ed. Rio de Janeiro: Elsevier, 2016.

LAKATOS, E.M. Metodologia do trabalho científico: procedimentos básicos, pesquisa bibliográfica, projeto e relatório, publicações e trabalhos científicos. 7. ed. 6. reimpr. São Paulo: Atlas: 2011.

LÉLIS, E.C. Administração de Materiais. São Paulo: Pearson Education do Brasil, 2016. Acesso em: 09 set.2016.

MARTINS, P. G.; AL, P.R.C. Administração de Materiais e Recursos Patrimoniais. 3. ed. São Paulo: Saraiva, 2011.

MELLO,L,T,C.et al.Análise do Lead Time nos Processos Logísticos de uma Rede Varejista de Flores. Produção onLine, v.16. n.4. 2016

PICININ, C.T.; KOVALESKI, J. L.; PEDROSO, B. Indicadores do transporte de carga: um estudo de caso na região dos Campos Gerais - PR. Nucleus, Ituverava, v. 8, n. 2, nov. 2011. ISSN 1982-2278. Disponível em: <http://www.nucleus.feituverava.com.br/index.php/nucleus/article/view/552〉. Acesso em: 26 aug. 2019. DOI 10.3738/1982.2278.552 .

PIRES, S.R. I. Gestão da Cadeia de Suprimentos: Conceitos, estratégias, práticas e casos. 2. ed. São Paulo: Atlas, 2011.

SLACK,N; CHAMBERS,S; JOHNSTON,R. Administração da produção. São Paulo. Atlas, 2002.

VIANA, J.J. Administração de materiais: um enfoque prático. São Paulo: Atlas, 2012. 